\title{
Suicides au travail : un drame de la conscience professionnelle?
}

Suicide at work: A drama of professional awareness

Yves Clot

\section{(2) OpenEdition}

\section{Journals}

Édition électronique

URL : http://journals.openedition.org/activites/716

DOI : 10.4000/activites.716

ISSN : 1765-2723

\section{Éditeur}

ARPACT - Association Recherches et Pratiques sur les ACTivités

\section{Référence électronique}

Yves Clot, «Suicides au travail : un drame de la conscience professionnelle? », Activités [En ligne], 10-2 | Octobre 2013, mis en ligne le 15 octobre 2013, consulté le 01 mai 2019. URL : http:// journals.openedition.org/activites/716 ; DOI : 10.4000/activites.716

\section{(9) $\odot \Theta \Theta$}

Activités est mis à disposition selon les termes de la licence Creative Commons Attribution - Pas d'Utilisation Commerciale - Pas de Modification 4.0 International. 


\title{
Suicides au travail : un drame de la conscience professionnelle ? \\ Yves Clot
}

Équipe Psychologie du travail et Clinique de l'activité Centre de Recherche pour le Travail et le Développement CNAM, 41 rue Gay Lussac, 75005 Paris

yves.clot@cnam.fr

\begin{abstract}
Suicide at work: A drama of professional awareness. The article proposes a working hypothesis on suicides in the workplace. Based on Vygotsky's work on activity theory and on G. Canguilhem's analysis of consciousness, it discusses the results of the sociology of suicide. It outlines the ongoing debates in work psychopathology. It develops the idea that suicides at work are dramas of work ethics and professionalism. The confusion between collective consciousness and self-consciousness must be studied to achieve a better understanding of the relationships between the individual and the organization of work which are at the heart of the problem.
\end{abstract}

\section{KEYWORDS}

suicides in the workplace, dramas of work ethics and professionalism, work psychopathology, Vygotsky.

Cet article a un statut un peu particulier. Il ne présente pas de résultats de recherche. Il est plus modeste et plus ambitieux à la fois. Il propose une hypothèse de travail qui tient compte d'un côté de la difficulté à rassembler, pour le moment, les données cliniques suffisantes pour instruire complètement le dossier et, de l'autre, des premiers travaux de références publiés en la matière.

Pour fixer un point de départ, on retiendra un exemple : au mois de novembre 2012, un guichetier de 55 ans s'est pendu dans un bureau de Poste à La Fère dans l'Aisne. Il a laissé un message envoyé par mail quelques minutes avant son suicide à deux cadres de La Poste et à une collègue. Nous reproduisons ici ce message rendu public par le guichetier lui-même.

\section{Bonjour,}

J'ai attendu jusqu'au dernier moment un vrai message d'espoir, un qui montrerait enfin un peu de reconnaissance du travail que j'ai effectué à La Fère, rien, rien du tout. Au contraire, pas de bouée pour celui qui se noie à cause des décisions d'une hiérarchie aveugle, juste quelques coups de bâton pour l'éloigner du bord. Cela ne fait que confirmer que Soutienpostier n'est qu'une façade. Ce n'était pas un rendez-vous avec l'assistance sociale ou le docteur qu'il fallait mettre en place, c'était juste s'intéresser à tout ce que j'avais mis en place pour la gestion du back-office de La Fère, et me dire en face que ce n'était que de la merde. Mais bon, ça y est, le problème est réglé.

Bien sûr, cette lecture provoque un embarras définitif. Le dernier mot est dit par cet homme sans qu'on puisse prétendre le comprendre. Et encore moins lui faire dire ce qui nous arrange. Mais cet ultime message est aussi une bouteille à la mer. Il ne règle rien pour ceux qui restent, bien au contraire. On pourra toujours parler d'un conflit de valeurs dans le travail. C'est indécidable. Ce qui l'est moins c'est la valeur du conflit sollicité - à défaut de l'échange - sur ce qui est fait. Ce message reste un appel à ce que quelque chose soit dit, en 
face, sur le travail réalisé. Ce qui a été mis en place dans le travail réel par ce guichetier appelle discussion et ne peut être supplanté, au moins pour lui, par la mise en place d'une écoute psychologique. La presse a rapporté, après le drame, que la hiérarchie de ce guichetier le jugeait «pointilleux » et a parlé, comme c'est souvent le cas, de "problème de reconnaissance ». On notera seulement que le message est plus précis et, en un sens, plus concret. Il demande « un peu de reconnaissance du travail » à propos du back-office.

On peut penser bien sûr que les raisons données à son acte par ce guichetier ne suffisent pas à l'expliquer. Nous n'avons aucun moyen d'en juger. Mais on peut aussi remarquer, sans pouvoir inférer davantage de la situation rapportée ci-dessus, que l'usage de ce « genre » de raisons est systématiquement au rendez-vous des «explications» laissées par ceux qui mettent fin à leurs jours, soit sur le lieu de travail, soit à l'extérieur en laissant des messages explicites à leurs proches. On cherchera donc à mieux les comprendre comme telles, sachant que l'un des acquis des travaux en psychopathologie du travail, corroborés par l'expérience de l'entourage professionnel et familial, est précisément que «ceux qui se suicident au travail se recrutent parmi les hommes et les femmes qui se sont le plus engagés dans le travail » (Dejours, 2007, p. 2). Ils sont donc, très fréquemment, intensément « investis » dans leur tâche, dans l'attente d'une reconnaissance tangible de leur activité par la hiérarchie et ce, d'autant qu'ils sont généralement des professionnels confirmés. Pour le dire dans un vocabulaire de tous les jours, ils sont connus autour d'eux pour faire preuve de beaucoup de conscience professionnelle.

\section{1.- Un problème de conscience ?}

À ce point, il semble indispensable de prendre plus au sérieux qu'on ne l'a fait jusqu'ici dans la littérature ce «concept quotidien » de conscience professionnelle. Il permet à beaucoup de travailleurs de marquer leur attachement au travail. On se donne rarement la peine de le définir au-delà de son usage spontané, saturé d'expériences concrètes, utile pour se faire comprendre à la fois entre collègues et par les hiérarchies. C'est comme un mot de passe ou un trait d'union chargé de sous-entendus multiples entre connaisseurs. Mais il est impossible d'en percer le sens de façon précise de l'extérieur à qui veut comprendre sa portée générale, son usage transversal à beaucoup de milieux professionnels. Pourtant évoquer la conscience professionnelle est une manière de parler à la fois de ce qu'il y de plus subjectif, de plus intime dans le travail, et d'une histoire sociale qui n'est pas seulement celle de chacun. Le langage ordinaire et ce qu'il dit de l'expérience concrète nous placent devant l'une des questions les plus difficiles de la psychologie. Si, comme le notait Vygotski en 1929, «l'individuel chez l'homme n'est pas le contraire du social, mais sa forme supérieure » (2004, p. 236), quand de tels drames personnels surviennent comment jouent ces relations de l'individuel et du social ? La question du suicide au travail ne nous laisse guère le choix de reprendre le problème. C'est pourquoi on se propose d'explorer comment la psychologie du travail peut aborder ce qu'on appellera ici des drames de la conscience professionnelle. Pour le faire, on aura recours à deux sources théoriques qui nous conduiront à suivre le chemin suivant : de la question de la conscience comme telle à la question du travail en société puis de celle-ci à la question du suicide.

$\mathrm{Au}$ regard de ces deux sources théoriques, on verra que la conscience ne peut pas être comprise comme une sorte de « solo du moi ». La première de ces références est celle, déjà utilisée, à Vygotski qui définissait la conscience tout court comme « un contact social avec soi-même » $(2003$, p. 91). On verra que c'est particulièrement vrai pour la conscience professionnelle. La deuxième nous pousse dans la même direction. Avec Canguilhem, la conscience n'est pas non plus le fantôme de la vie intérieure. Elle ne va pas nue, mais toujours habillée : " pas de pensée sans apprêt. Point de conscience sans reprise, c'est-à-dire sans métier » (2011, p. 323). Elle ne peut être étudiée que dans ses œuvres. Le « connais-toi, toi-même » de l'introspection n'a guère de sens. Mieux vaut éprouver ce dont on est capable 
en situation. Et finalement, non sans humour, Canguilhem conclut par cet éloge de l'activité : «il n'y a de conscience que professionnelle » (p. 323).

C'est pourquoi, quand il s'apprête à aborder la question du suicide en 1939 dans un Traité de logique et de morale récemment réédité (2011), il le fait contre l'illusion d'une vie intérieure retranchée de la vie sociale et exposée seulement à la fragilité d'un sujet: «la vie n'a de valeur, comme toute chose, qu'en rapport avec ce qui la dépasse et l'enrichit» (p. 873). Et « la valeur durable de la personne ne peut se résoudre qu'en plaçant le principe de cette valeur en dehors même de l'individu et d'ailleurs aussi d'une société quelconque » (p. 873). La dignité se trouve dans le rapport de mon activité personnelle avec l'idée d'humanité en général. Si je concours à donner un sens à cette idée, «j'ai déjà quelque honneur ou quelque dignité. Si je ne fais rien dans ce sens, et si au contraire je contribue par ma veulerie à rabaisser l'idée d'humanité en moi, le mot d'honneur n'a aucun sens pour moi » (idem, p. 871). Vivre, pour l'homme, c'est toujours avoir le sentiment d'exister pour autre chose que lui-même.

Il y a toujours dans un suicide une rupture avec ce sentiment d'exister au dehors et au-delà de soi. C'est plus que la rupture avec un milieu : «en finir avec la vie, fût-ce au fond d'un désert, c'est en finir avec la société » (p. 871). Il n'y a pas bien sûr d'apologie du suicide chez Canguilhem mais malgré tout un rappel historique: la mort volontaire comme comportement de «sécession» dans les cités grecques soumises à la domination macédonienne face à un système politique sur lequel l'individu ne pouvait plus exercer d'action ni de contrôle. Contre le suicide, note-t-il, on invoque souvent la lâcheté ou la désertion du devoir social : «Pour beaucoup le suicide est une impolitesse, une incongruité, étant un départ sans demande préalable de congé » (p. 873). Pourtant, «il est humain, c'està-dire respectueux de la valeur, de s'abstenir de juger le suicide » (idem). Personne n'a pu vouloir sa vie en tant que telle en décidant de sa naissance: "la seule initiative que l'individu puisse prendre concernant sa vie c'est de la finir. Le suicide est donc la manifestation de puissance limitée d'un être dont l'engagement dans la vie, inexplicable en fait, devient pour lui injustifiable en droit» (p. 872). Et Canguilhem de conclure en une phrase qui marque bien le risque d'une défaillance toujours possible du contact social avec soi-même: "Supposons, sans grande hardiesse, la persécution des minorités raciales ou politiques devenue la règle sur toute la terre, la seule planète jusqu'à présent accessible à l'espèce humaine, et demandons-nous si nous pourrions encore prétendre à juger la résolution de ceux qui préfèreraient devoir la mort à leur courage plutôt qu'à la férocité d'autrui. Dieu, s'il admet les pogroms, pourquoi n'admettrait-il pas aussi les suicides ?» (p. 874).

Devant ces persécutions que Canguilhem désigne comme une «abjection sociale», l'initiative d'en finir peut suivre la décision de ne pas accepter l'injustifiable rabaissement de l'humanité en moi. Non seulement pour moi-même, mais parce ce que ce qu'on me fait, on le fait au genre humain tout entier. Si la conscience est un contact social avec soi-même, elle est donc aussi un contact très personnel de la société avec elle-même. Quand ce contact simultanément personnel et social provoque un court-circuit aussi grave que les suicides en milieu professionnel c'est aussi bien le rapport de la société avec elle-même qui mérite attention; et pas seulement - même si nécessairement - le rapport du sujet à lui-même.

\section{2.- Le point de vue des sociologues}

C'est d'ailleurs la thèse défendue par C. Baudelot et R. Establet : «Ce n'est pas la société qui éclaire le suicide, c'est le suicide qui éclaire la société » $(2006$, p. 17 ; voir également Barlet \& Marichalar, 2012). Pour mieux définir ce que nous entendons par drame de la conscience professionnelle on résumera très vite les résultats obtenus pas les deux sociologues, redevables à Durkheim, mais à distance de lui : "Durkheim situait la causalité sociale du suicide à l'étage des interactions individuelles pour le suicide par défaut 
d'intégration et, dans le cas du suicide anomique, il l'inscrivait dans le domaine de la psychologie individuelle : l'individu perd alors les repères qui lui permettaient d'ajuster ses comportements aux règles en vigueur dans son groupe social et de régler ses désirs sur les possibilités de les satisfaire (...), mais il en reste à un niveau très général ; jamais il ne prend en compte les conditions matérielles d'existence et de travail des individus » (2006, p. 201). Pourtant à partir de l'enquête quantitative, Baudelot et Establet établissent un double résultat dans l'histoire. Conformément à la thèse de Durkheim d'abord, le développement de l'individualisme qui est à la fois l'une des conséquences et l'une des conditions du développement économique capitaliste est bien porteur, selon eux, de facteurs favorables au suicide. Mais ce premier résultat ne doit pas en cacher un autre de sens inverse : «Le développement dans les pays riches, et tout particulièrement dans la sphère professionnelle de valeurs liées à la réalisation de soi et à un individualisme créatif, permet d'atténuer, voire de contrecarrer, les effets négatifs du premier individualisme. Un retournement identique s'est opéré dès la première moitié du XXème siècle avec l'urbanisation. Les grandes villes multipliaient les risques de suicide au XIXème siècle, elles le diminuent au XXème siècle. En s'enrichissant, les sociétés développées ont su inventer de nouvelles façons d'être ensemble, à la ville et au travail » (2006, p. 104). Certes, le primat de la personne sur le groupe engendre bien d'abord une hausse du suicide, mais «plus on associe dans un pays donné le travail à la réalisation de soi et à la sociabilité, plus on efface sa pure dimension de gagne-pain, et plus le taux de suicide baisse» (p. 101). Plus le «sentiment de faire des choses qui restent » (p.99) prévaut avec le sentiment d'appartenir à un ensemble qui dépasse chacun pour la réalisation de soi (p. 197), plus le taux de suicide baisse.

On comprend alors l'intérêt de ces sociologues pour les métamorphoses actuelles du travail : «Largement ignoré par Durkheim dans ses analyses du suicide, l'univers du travail représente aujourd'hui un domaine stratégique où se noue, de façon contradictoire, tout un ensemble de relations entre l'individu et la société, et où l'individu joue gros » (p. 104). Et les changements du travail sont loin de seulement prolonger les tendances du passé. Chaque travailleur joue gros aujourd'hui sous la pression des nouvelles formes d'organisation où on l'incite à s'impliquer personnellement au risque de transformer «la réussite ou l'échec en verdict existentiel» (p. 105). En cas de revers, la responsabilité professionnelle vire à la culpabilité personnelle surtout quand le dégrisement arrive et que les travailleurs très impliqués sont conduits à penser que n'importe qui aurait pu faire leur travail à leur place. C'est peut-être supportable pour les salariés protégés par leur capital social et culturel pour qui rien ne vaut la vie. Mais, pour les moins bien lotis, le cumul de conditions de travail et d'existence très dégradées, génératrices d'angoisse persistante, peut rendre insupportable ce dégrisement et « la mort peut au contraire être préférée à une vie d'enfer » écrivent Baudelot \& Establet (p. 207) dans des termes rappelant ceux de Canguilhem.

Mieux, les sociologues mentionnent les recherches ethnologiques de Dorothy Ayers Counts sur les suicides de protestation chez les femmes Lusi de Nouvelle-Guinée. Là, contre les mauvais traitements conjugaux et beaux-parentaux, le suicide prend place dans la gamme des moyens de résistance que les femmes utilisent pour parvenir, paradoxalement à leurs fins. Elles font en sorte, par un acte souvent public, que les motifs en soient connus de tous : «l'intention vindicative est clairement affirmée ; et les personnes visées sont explicitement désignées » (p. 231). Ils élargissent encore le spectre à l'aide de l'exemple paradoxal de la Chine qui fait exception à la tendance générale. C'est le seul pays au monde où les femmes se suicident plus que les hommes : «Aujourd'hui comme hier, le suicide demeure une des armes de protestation dont disposent les femmes chinoises pour lutter contre le mépris traditionnel attaché à leur sexe et à la violence de leur belle-famille » (p. 240). Et, au total, en pensant d'abord à la brusque montée du suicide des jeunes dans nos sociétés européennes, Baudelot \& Establet nous proposent de «prendre en compte une dimension anthropologique, absente chez Durkheim et même chez Freud : l'orientation vindicative. Peut-être n'est-elle pas absente du suicide dans les sociétés industrielles » (p. 234). Et il faut bien convenir que 
cette hypothèse mérite d'être prise très au sérieux dans le champ de l'analyse des suicides au travail même si «une profession, au même titre qu'un niveau de revenu, ne saurait constituer, à elle seule, un facteur et encore moins une cause de suicide» (p. 185). Cette hypothèse d'une orientation vindicative du suicide au travail n'est pas celle qui est classiquement retenue dans la littérature sur la question. Elle a bien sûr le gros avantage de questionner l'organisation sociale du travail subordonné au lieu de pousser l'action à « éclairer » la fragilité des travailleurs.

\section{3.- Débats dans la psychopathologie du travail}

Mais nous voudrions la confronter aux dimensions plus psychologiques propres au drame de la conscience professionnelle évoqué ci-dessus. La dimension psychique de la question a été explorée par C. Dejours depuis longtemps. Ses travaux font référence en la matière. Pour C. Dejours et $\mathrm{F}$. Bègue, en appeler à la vulnérabilité psychologique pour rendre compte du suicide au travail est tout à fait insuffisant : "si la cause dernière était de ce côté, on ne comprendrait pas pourquoi aujourd'hui nombre de suicides sont commis par des sujets qui ne présentent aucun symptôme prépathologique et affichent d'excellentes performances professionnelles » $(2009$, p. 28). C'est même leur grande aptitude à s'adapter aux contraintes de travail qui leur permettrait de "s'engager pleinement pour l'entreprise et d'atteindre des performances de travail over the target, au-dessus de la cible » (ibid, p. 28). Le suicide est alors provoqué par une disqualification et une «disgrâce inattendue » qui vient dégriser les illusions d'un engagement « corps et âme » (p. 41). Mais cette disgrâce managériale ne suffit pas pour rendre compte de la multiplication des suicides au travail. Cette multiplication « résulte principalement de l'expérience atroce du silence des autres, de l'abandon par les autres, du refus de témoigner des autres, de la lâcheté des autres » (p. 45). L'humiliation au travail ne suffit pas, c'est la « trahison des collègues et des proches » qui emporte finalement les travailleurs concernés survivants dans des communautés professionnelles réduites à un regroupement factice pour le travail. C. Dejours insiste sur les risques d'une «convivialité stratégique » qui se présente comme une véritable «condition sociale » surtout chez les cadres (2010, p. 150).

Pourtant, pas de suicide au travail sans «faille» personnelle selon C. Dejours. Mais le paradoxe, selon lui, est que cette vulnérabilité personnelle explique aussi «la force de l'implication humaine dans le travail » (p. 158). À partir de l'analyse approfondie d'un cas de suicide, C. Dejours note qu'une grande «puissance de travail » peut être obtenue au prix d'une «exigence impitoyable» à l'égard de soi-même chez tel professionnel et que l'entreprise est capable d'utiliser cette «rigidité personnelle» (pp. 154-158) ${ }^{1}$. On trouvera déjà chez L. Le Guillant, l'un des fondateurs de la psychopathologie du travail, une analyse devenue classique des risques que peut faire courir à certains salariés cette « exigence morale rigoureuse » qui peut «dramatiser» la situation de travail (2006, pp. 184 et suivantes). Et, du coup, on peut comprendre l'analyse clinique de Dejours pour l'histoire personnelle qu'il rapporte: «Sa raideur morale était aussi ce qui faisait d'elle une professionnelle particulièrement brillante et appréciée » (p. 158). Jusqu'à son suicide, «sa performance de travail est restée jusqu'au bout au plus haut niveau » (p. 161). On comprend alors que «c'est à cause de cette vulnérabilité que nous sommes parfois capables de performances professionnelles remarquables $\gg($ p. 158).

Mais faut-il, pour autant, en conclure que «l'entreprise exploite nos vulnérabilités et qu'il n'y a peut-être rien de condamnable à cela, puisque, dans certains cas, nous pouvons aussi en être bénéficiaires » (p.158) ? Ou encore «qu'aucun travail de qualité n'est possible sans l'engagement de la subjectivité tout entière»? (p.157). Ou même que cet engagement

1 Voir aussi l'analyse datant déjà de plus de 20 ans, d'une tentative de suicide au travail par B. Doray (2011, p. 156). 
implique que le sujet, pour s'affronter au réel, « accepte de se faire habiter tout entier par son travail»? (p. 157). N'y a-t-il pas là justement une conception de la performance très discutable ? Est-elle dans ce cas de si « haut niveau »? Peut-on même aller jusqu'à dire que ceux qui se suicident au travail se recrutent « parmi les meilleurs » (Dejours, 2007, p. 2) ?

Ce point a été discuté par P. Molinier à la suite d'une enquête de psychodynamique après un suicide lié au travail (2009) : sont-ce les «meilleurs » qui se suicident ou ceux qui ont le plus de mal à s'autoriser une souplesse à l'égard de la fascination pour la performance apparente et pour les buts factices qu'elle organise? Voir les choses ainsi a des conséquences importantes déjà relevées (Lhuilier, 2009). En tous cas, la question se trouve, selon P. Molinier, deux fois déplacée : du côté des conditions collectives qui autorisent cette souplesse, mais aussi du côté des configurations psychiques individuelles: la créativité «implique une certaine souplesse psychique, de pouvoir renoncer à des conceptions idéalisées du travail et de soi-même, donc un certain rapport au réel et à l'échec » (2009, p. 51). On peut encore le dire autrement : «Qui décide de qui sont les meilleurs?» (Ibid, p. 50). On ajoutera : peut-on jamais être vraiment «bénéficiaire»d'une conception de la performance réclamant d'être habitée entièrement par son travail, impliquant comme une qualité indispensable une certaine « raideur », pour parler comme Dejours. Rien n'est moins sûr. On peut rappeler ici encore l'analyse classique de Le Guillant, à propos du travail des téléphonistes, qui montrait dès les années 1950, que leur «nervosité personnelle » était paradoxalement une maladie nécessaire et fonctionnelle pour l'organisation du travail. Cette «nervosité » était une «aptitude» paradoxale, une aptitude à supporter l'intensification anormale du travail (2006, p. 91 et suivantes).

Et il semble bien que, plus d'un demi-siècle après, la conception dominante de la performance reste profondément «anormale » avec les conséquences qu'on examine ici (Lhuilier, 2011). Se sentir - à tort ou à raison - contraint à un engagement «corps et âme » dans le travail est profondément «anormal ». Au-delà du fait que c'est là le symptôme d'une organisation du travail qui refoule la performance réelle sous l'inflation chiffrée d'une performance apparente, avoir à «se donner tout entier» est peut-être le genre de situations où se noue justement ce qu'on a appelé plus haut le drame de la conscience professionnelle: ce drame si personnel et tellement social à la fois qui se noue derrière le simulacre du travail bien fait. Qu'est-ce que cette «raideur» d'abord vue comme une aptitude et qui peut pourtant ensuite se refermer comme un piège sur les sujets? En quoi peut-on parler à son propos d'un drame personnel de la conscience professionnelle? N'y aurait-il pas une sorte de rigidité sociale à sa source ? Si oui, de quel genre ?

\section{4.- Conscience collective et activité}

Ici il faut prendre au sérieux la formulation de Vygotski en la transposant : la conscience professionnelle est un contact social avec soi-même. Commençons donc par le contact «social» en question, c'est-à-dire par la conscience professionnelle comme conscience collective avant même d'être la conscience professionnelle de tel ou tel travailleur. On connaît les propriétés de cette dernière : avoir sa conscience pour soi dans ce qu'on fait, le faire dans les règles de l'art, à tort ou à raison, mais au nom de ce qu'on croit juste.

Mais la conscience professionnelle - comme les règles de l'art - existe d'abord au plan social et collectif, dans un domaine, pour un métier, dans une organisation et même en dehors d'elle, pour les consommateurs ou les usagers par exemple. On pourrait spontanément la désigner à la manière dont Durkeim décrit les «représentations collectives » quand il étudie le fait religieux, comme un bagage que l'individu reçoit de la société, comme un ciment d'idées ou de manières de sentir dont les psychologies individuelles ne sont finalement que le support ; comme l'adhésion et le consentement à des valeurs professionnelles transmises. Voir les choses ainsi a l'avantage d'enraciner la conscience professionnelle dans l'ensemble des institutions sociales où elle est cristallisée. 
Mais c'est envisager alors cette conscience un peu comme un état mental collectif sédentaire, un patrimoine idéal à reprendre à son compte. En décalage avec ce mentalisme social, on regardera plutôt ici les significations collectives dans leur rapport réel avec les activités communes concrètes, à l'épreuve du travail ordinaire. Si les représentations collectives de la conscience professionnelle dans un métier sont bien sûr instituées, c'est pourtant le commerce quotidien entre les activités réelles qui les institue, les ré-institue ou encore les destitue. Autrement dit, les représentations collectives ne contiennent pas en puissance les activités collectives. Elles ne les expliquent pas. La conscience professionnelle non plus. Ce sont plutôt les activités collectives qui s'expliquent, aux deux sens du terme, avec les représentations de la conscience professionnelle pour faire face au réel. Quand ces activités ne gardent pas vivantes les représentations collectives grâce aux objections qu'elles leur proposent ces représentations peuvent s'appauvrir ou encore se nécroser.

La conscience professionnelle, comme la conscience tout court (Clot, 2003), n'existe pas comme un état mental, mais comme un rapport social réel. C'est seulement en mouvement qu'elle montre ce qu'elle est. Si nous la séparons de la vie pratique du travail, de l'activité effective, nous la privons de sa fonction principale qui consiste, dans l'action, à rester l'instrument pour franchir les obstacles du travail réel. Au travail, elle est exposée, sinon elle s'anémie. Laissée à elle-même la conscience professionnelle propre à un métier s'étiole. À la suite de Vygotski, on pourrait dire que l'arrachement de la conscience professionnelle au réel du travail est destructeur pour elle-même ; elle se nécrose, perd sa profondeur collective, subjective et sa mobilité, ses possibilités d'élargir et de renouveler ses ressources dans des contextes neufs et vivants ; pour tout dire elle meurt en tant que conscience professionnelle, car les représentations collectives vivent en dehors d'elles-mêmes, seulement quand elles restent orientées vers l'extérieur. Loin d'être le fond immuable de l'appartenance à un groupe professionnel, le ciment qui le fixe, elles sont un instrument de travail dont l'impact est à entretenir ensemble.

Comme conscience collective, la conscience professionnelle a donc besoin d'être régulièrement réveillée au contact du réel et, comme l'écrit Halbwachs, «en quelque sorte rechargée » dans l'activité collective, «comme pour y commencer une nouvelle carrière » (1955, p. 54-55). Assez loin de Durkheim, comme l'a bien vu H. Wallon (1971, p. 299), Halbwachs nous permet d'envisager la conscience collective non comme un monde à part qui s'entretient de lui-même et qu'il faudrait respecter, mais comme un mouvement fonctionnel : celui des représentations dans l'activité en cours qui les régénère, mais où elles peuvent aussi dégénérer. S'ouvrent ainsi les voies d'une discordance nécessaire à la pérennité et au développement de la conscience professionnelle collective. Elle peut s'engourdir si elle n'est pas mise régulièrement au travail. Elle se présente comme l'institution toujours à refaire d'une liaison exposée par nature aux risques de la tâche et de l'activité : la liaison fragile et potentiellement défunte entre les différents registres de la conscience professionnelle qui constituent autant de composantes de sa dynamique collective. Comment les décrire?

\section{5.- Détour par le métier}

Ici, il nous faut faire un détour. Dans les travaux en clinique de l'activité nous avons l'idée que le métier et même le travail ont une architecture en tension: au plus près du réel de l'activité - qui ne peut jamais être complètement anticipée - le travail se signale d'abord par le geste personnel - qu'il soit physique ou mental - propre à chacun et qui fait dire d'un tel qu'il a du métier. Mais il n'y a pas de solo de l'activité dans cette histoire personnelle. Dans l'exercice professionnel, on ne pense jamais tout seul même si, bien sûr, on pense par soi-même. Le geste personnel est toujours adressé aux collègues destinataires des efforts consentis. Le travail est à ce titre interpersonnel. Il vit donc - ou il meurt entre professionnels et en chacun d'eux dans la motricité des échanges personnels et 
interpersonnels sur ce qui est à faire ou à refaire, à dire ou à redire, sur ce qu'il aurait fallu faire et même sur ce qu'on aurait pu faire - et même bien faire - alors que l'activité est empêchée (Clot, 2008). Cette grammaire de l'action montre à quel point travailler est un acte de conjugaison. Le travail n'est d'ailleurs pas non plus tout entier dans le présent des activités partagées en situation. Ces dernières ont besoin d'antécédents parfois lointains qui permettent de se mettre au diapason de ce qui arrive : c'est l'histoire et la mémoire collective qui donnent à chacun le répondant pour agir dans le présent et «voir venir » le futur. Cette mémoire est transpersonnelle, personne n'en est propriétaire. Disponible pour tous quand elle existe, elle peut traverser les générations et chaque professionnel individuellement. C'est le clavier collectif des gestes et des mots sur lequel chaque sujet peut jouer sa petite musique, le genre professionnel à styliser par chacun (Clot, 2008). Institué, mais toujours potentiellement perdu, organisé par des sous-entendus ou des rébus, ce genre est un trait d'union qui risque toujours de s'effacer, s'il n'est pas refait dans le cours des activités interpersonnelles et personnelles.

Enfin, le métier n'est pas institué seulement dans la mémoire collective transpersonnelle. Il existe aussi, impersonnel, consigné et conservé dans les prescriptions officielles. Ce sont aussi bien des indicateurs standards de performance, des critères d'évaluation et de recrutement, des tâches, un statut d'emploi, des formations reçues, bref un autre monde que celui de l'activité réelle en situation ou de l'histoire collective implicite. Même si les prescriptions générales font leur une partie du travail générique implicite, elles sont l'appareillage le plus éloigné de l'activité concrète. Au contact de ce registre explicite de la tâche prescrite, chacun se sent sans doute interchangeable. Mais ce registre impersonnel est psychologiquement vital. On s'y mesure, on s'y retrouve ou on s'y investit. On en use, on s'y oppose ou on s'en détourne pour travailler. Mais quoi qu'il en soit, on n'y échappe pas (Clot, 2008).

Des conflits se faufilent partout dans cette architecture mobile de l'exercice professionnel, où « le travail bien fait » - à quoi on identifie souvent la conscience professionnelle n'est pas, en réalité, d'un seul tenant et obéit à plusieurs critères qui peuvent s'épauler, mais aussi très souvent s'opposer (Clot, 2010). Car le travail relie ou plutôt cherche à relier dans des discordances créatrices ou destructrices - ces quatre registres impersonnel, transpersonnel, interpersonnel et personnel. Toutes les variations sont possibles: par exemple, un travail personnel soigné auquel on cherche à se tenir peut se révéler être un obstacle dans le travail collectif interpersonnel acceptant de s'ajuster à des contraintes impersonnelles, occasionnelles, mais justifiées. Mais ce travail collectif en mode dégradé luimême peut aussi être en contravention durable avec le genre professionnel (Clot, 2008), affaiblissant à terme les ressources transpersonnelles de l'activité de chacun. Au risque de discréditer jusqu'à l'idée même de stylisation personnelle et de création, non sans dommage pour la vitalité de la prescription impersonnelle. Cercles vicieux et cercles vertueux du métier s'affrontent toujours dans des développements possibles ou impossibles du «travail bien fait » dont les destins ne sont pas écrits d'avance.

\section{6.- « Raideur » dans la conscience professionnelle collective}

On peut penser, et c'est maintenant ce que nous proposons de soutenir, que la conscience professionnelle collective épouse ces registres, elle aussi avec ses propres cercles vicieux ou vertueux en conflits. On la retrouve à l'œuvre, plus ou moins, dans la vie réelle et l'activité conjointe de chacun avec ses collègues; mais aussi dans la réserve des règles de l'art génériques disponibles entre eux au-delà même de la situation où ils se trouvent. On la découvre aussi - sans qu'on y pense assez - très loin de ce que chacun est justement en train de faire avec ses collègues, dans les procédures générales de la prescription où elle est souvent «arrêtée» pour commander, proscrire ou outiller. Le rapport entre les représentations collectives présentes sur ces quatre registres et l'activité en cours, qui les 
convoque pour fonctionner - et parfois dysfonctionner - ensemble, est le mouvement même de la conscience professionnelle collective. Leur concordance, par nature imparfaite, est éprouvée dans l'action. On comprend alors que la conscience collective en question ne soit pas toujours vivante pour les mêmes raisons ni au même endroit. Morte à un bout elle peut ressusciter à l'autre. Elle n'est nulle part stockée tout entière, ni dans l'activité de chacun, ni dans la tâche, ni dans le collectif. Vivante, elle transite partout sur des frontières fluctuantes. Morte sur un registre elle expose l'ensemble. Quiconque prétend l'incarner exclusivement la manque et l'usurpe. C'est un point de fuite que ne peut s'approprier nul individu, nulle entreprise, nul collectif. Aucun n'en a le monopole.

Le paradoxe de ce déséquilibre irréductible de la conscience professionnelle collective devrait soumettre tous les protagonistes concernés aux exigences de la modification. La santé en dépend : à charge pour les professionnels dans l'organisation de maintenir cette conscience en tension, c'est-à-dire en activité, au contact des surprises du réel, de la lester par ce qui la tient : la qualité du travail à jamais discutable. Faire son travail c'est peut-être réussir ou échouer à prendre soin de cette architecture sociale de la conscience professionnelle. Car ses ressources ne sont pas entreposées toutes au même endroit et méritent pourtant d'être entretenues partout.

Ne soyons pourtant pas naïfs. Dans cette architecture, tout est lié, mais tout est souvent délié par des antagonismes irréductibles, réels ou supposés. Par exemple, au travail, le sentiment transpersonnel de vivre la même histoire peut s'éteindre en laissant libre cours aux querelles de personnes. Un métier sans répondant transpersonnel peut ainsi dégénérer en antagonisme dévastateur entre un exercice personnel solitaire et subordonné et des injonctions impersonnelles coupées du réel. Une vraie dépersonnalisation du travail s'en suit alors, ce qu'on regardera comme un déchirement de la conscience professionnelle collective. C'est au-devant de cette déliaison menaçante que se porte habituellement l'action en clinique de l'activité (Kostulski, Clot, Litim, \& Plateau, 2011). Mais la déliaison peut prendre aussi une forme inverse qui tend aujourd'hui à s'imposer et qu'on peut retrouver jusque dans les drames personnels de la conscience professionnelle : la confusion entretenue des registres entre eux, leur amalgame toxique. Comme si la conscience professionnelle - comme institution collective alors nécrosée - pouvait fusionner entre eux et sceller sans résidus ces registres dans une conscience collective soudée. On trouvera alors tous les exemples de système collectifs défensifs alimentés par des fonctionnements institutionnels aussi bien que personnels, complices des cercles vicieux les plus dangereux à la fois pour la santé et pour l'efficacité (Fernandez, 2009; Molinier, 2009 ; Scheller, 2002).

Dans notre perspective, le «contact social » en question n'est donc pas sans risque quand il devient homogène. À l'inverse, au principe même de la liaison, il y a la différenciation des registres et la circulation possible entre eux. Il y a même le conflit nécessaire entre ces registres de la conscience professionnelle comme ressource pour le développement de l'expérience. Chacun d'eux est le moyen potentiel, dans le travail d'organisation collectif (Maggi, 2011; de Terssac, 2011), de «prendre du recul» sur les autres, force de rappel contre l'enveloppement de la conscience professionnelle sur un seul registre ; contre les «idées fixes» de certaines de ses représentations collectives sur lesquelles elle peut s'arrêter. Quand le jeu des différenciations et la polyphonie des registres impersonnel, personnel, transpersonnel et interpersonnel de la conscience professionnelle se trouvent bloqués dans une organisation du travail qui parle d'une seule voix ; quand l'activité vivante se heurte par exemple à l'impossibilité effective de délier ces «consciences » différentes pour les relier aux objets ou aux destinataires réels de l'action en cours, cette activité est déréglée, empêchée ou emballée (Lhuilier, 2011, p. 200). Le travail d'organisation de l'activité en train de se faire s'expose à une conscience professionnelle collective d'un seul bloc, amorphe, en quelque sorte. Au lieu de pouvoir y puiser la mobilité inter-fonctionnelle requise par l'action quand il faut «faire le tour» d'un dilemme réel, les activités individuelles et collectives doivent se ranger derrière un seul point de vue. Ce genre de 
situations déchire la conscience collective et alourdit l'action en la tenant à l'étroit. Elle accapare les énergies et les initiatives en les enveloppant dans une conscience professionnelle torpide qui les arrête, les encercle, les détourne du développement réalisable au lieu de leur prêter le luxe possible de son agilité. S'en suit une «raideur » sociale qui paralyse le travail «bien fait », la performance réelle, sous les apparences du chiffre. Il s'agit là d'une conscience collective immobilisée, embaumée dans les formalités de la mesure dont l'activité doit réussir à s'affranchir, souvent à un coût démesuré. En particulier pour la santé quand une performance impersonnelle asservie débouche sur un professionnalisme faussé.

\section{7.- Monologue social et dialogue intérieur}

Car tout est là : à se prêter à ce jeu de l'indifférenciation dans la conscience professionnelle collective, on y risque sa santé mentale. Car on ne s'y reconnaît pas vraiment. Et l'illusion de pouvoir s'y donner «corps et âme» sans renoncer à soi-même finit par avoir un prix exorbitant. Il faut faire une croix sur le contact très personnel et même intime que chacun entretient dans son histoire propre avec la société, bien au-delà du travail.

Le drame est là : se donner « tout entier» au travail expose dangereusement la santé mentale. Mais ici il faut faire attention : c'est un certain type de contact social qui l'induit, celui que nous avons cherché jusqu'ici à cerner : un contact social «refroidi» ou encore irréel qui devient la source très dangereuse d'un «contact personnel» désaffecté avec la société. La conscience professionnelle collective engage certes un contact social avec soi-même. Mais selon la nature du contact social qui domine dans l'organisation c'est un genre bien particulier de contact subjectif avec la société qui est exigé : un contact drastiquement simplifié et unilatéral avec soi-même dans l'activité, guetté par la dépersonnalisation : on est alors actif sans se sentir actif. Le désaveu organisationnel d'une distance possible entre le personnel et l'impersonnel et la défiance à l'égard du travail collectif transpersonnel, qui, tous deux, compliqueraient la vie de l'organisation «idéale» par des «problèmes de conscience superflus », compromettent l'originalité irréductible de ce contact subjectif. Il le nivelle.

La vitalité de la conscience professionnelle de chaque sujet réclame un dialogue intérieur avec les mots de la conscience collective sur tous les registres en mouvement, une lutte avec eux pour $\mathrm{y}$ engager ses propres intentions et accents et, finalement, en prendre personnellement possession. Il est pathogène qu'un monologue impersonnel s'impose comme seul «contact social» avec soi-même. Bien difficile alors d'y mettre vraiment du sien : un contact social rendu indiscutable et finalement «cassant» par le déni de toute conflictualité professionnelle de la conscience collective peut «court-circuiter » la motilité de l'histoire subjective. Alors elle est sollicitée d'un seul bloc. Le monologue social assourdit le dialogue intérieur. Il convoque plutôt chacun à s'abandonner à une conscience discrétionnaire et monologique qui, à la première déconvenue professionnelle, exposera pourtant dramatiquement le sujet. Celui-ci se fait ou se défait, nous dit Vygotski (1997) en employant à son propre égard les formes de conduites que les autres ont d'abord employées envers lui. Lorsque ces dernières relèvent du monologue social, elles se retournent «avec lui » contre une vie subjective soumise du coup à une alternative : se simplifier radicalement jusqu'au soliloque ou trouver ailleurs, dans le travail ou en dehors, avec ses ressources propres, de quoi conjurer ce drame de la conscience professionnelle. Et rien ne garantit, on le sait, que ce soit possible pour tous.

Mieux vaut que la vie sociale au travail reste un «coupe-circuit» salutaire contre les subjectivités d'un seul bloc. Ce «coupe-circuit » est à entretenir ensemble. Les institutions de l'entreprise sont comptables, au nom même de la qualité réelle du travail, d'une résistance à la tentation monologique. L'organisation du travail doit rester discutable pour ne pas réclamer à chaque sujet de se livrer en totalité, en faisant la croix sur ce qui l'en protège : les dialogues intérieurs de sa conscience professionnelle propre. 
On se souviendra ici de la contribution de l'un des inspirateurs majeurs de la psychopathologie du travail, F. Tosquelles. À l'hôpital psychiatrique, il a promu dans les années 1960 une ergothérapie qui tournait par avance le dos à celle que nous connaissons aujourd'hui. Il insiste : quand l'hôpital comme institution vit ses conflits inter-fonctionnels et peut les assimiler, chaque malade peut « investir ceux des siens auxquels il s'était dérobé et par là, les résoudre » (Tosquelles, 1984, p. 84 ; Clot, 2009). Cette remarque porte loin audelà de l'hôpital psychiatrique. Elle signale que c'est la qualité de la conflictualité sociale entretenue dans l'institution qui règle ou dérègle la conflictualité interne du malade. On peut en tirer l'idée plus générale qu'une vie sociale qui n'offre plus de conflictualité externe suffisante dissipe l'énergie psychique des sujets. La conflictualité interne de la vie psychologique, le dialogue intérieur à la base de sa dynamique psychique - elle aussi interfonctionnelle - ne peut donc se maintenir sans relais social l'alimentant en énergie conflictuelle, pour parler comme G. Laval (2002, p. 69). Telle semble être l'idée-force de Tosquelles. Il a souvent donné de beaux exemples d'interposition institutionnelle de ce type dans l'activité propre des malades.

Ajoutons alors qu'une organisation du travail qui, au contraire, déniant ses logiques contradictoires, croit pouvoir éliminer sa conflictualité propre au nom d'une performance factice imposée sans débat et sans arbitrage, détruit l'intercalaire social indispensable au dialogue intérieur de chacun. En abrasant les disparités de la conscience professionnelle collective, elle abaisse le seuil de tolérance de la conscience professionnelle de chaque sujet à ses propres conflits. Chaque travailleur «en son âme et conscience» définit grâce à son passé à lui - fait à plusieurs bien sûr - la possibilité qu'a une situation de l'affecter (Malrieu, 2013, p. 137). Cette originalité est précieuse pour la santé en permettant à chacun d'être comme les autres à sa manière singulière (Molinié, Gaudart, \& Pueyo, 2012). Or, au lieu de garder justement les contradictions et les possibilités de chacun ouvertes sur les différends légitimes de la conscience collective, l'organisation du travail les referme sur leur occlusion. Tout en pâtit, aussi bien l'efficacité réelle que la santé. Mais on remarque alors que c'est sous la forme d'une causalité indirecte, celle qui fait agir l'organisation du travail par un détour : elle prive d'énergie la marche originale de chaque conscience professionnelle singulière en la coupant des pulsations «normales » du social.

\section{8.- Le nœud du drame}

Un travail de qualité durable n'est pas possible sans pouvoir «prendre du recul » avec soimême à l'aide d'une organisation du travail qui encourage l'exercice en assumant ses propres battements. L'anesthésie de la conscience professionnelle est le contraire de cela. Loin d'être la meilleure des adaptations, la fusion des registres personnels et impersonnels, sans force de rappel, joue avec le feu. Les dégrisements du réel peuvent devenir des épreuves insurmontables pour ceux que cette confusion a subordonnés. Dejours l'a bien vu : devant l'échec, quand la disgrâce arrive avec la disqualification par la hiérarchie de l'engagement opiniâtre dans le travail, on se sent désavoué : «il faut admettre que l'on n'a pas été seulement trompé par l'entreprise, mais qu'on s'est trompé soi-même » (2010, p. 155) en constatant que les renoncements auxquels on a consenti, parfois contre «les voix de sa conscience », n'ont servi à rien. Alors, d'abord le travail réalisé qu'on croyait inattaquable, puis soi-même et les autres et enfin l'entreprise qu'on tenait pour une seule et même chose se révèlent inaccordables. La fausse unification qui avait prévalu et qui a fini par dicter la conduite du sujet en refoulant les divisions et les interférences des registres se fissure. Il faut revoir alors toute sa vie prise au piège de l'indistinction ${ }^{2}$. Sans frontière entre eux, les

2 Baudelot et Establet ont éclairé la différence sociale entre les hommes et les femmes sous cet angle. Ils marquent combien les risques de ce piège sont plus grands chez les hommes (2006, pp. 237/238). 
registres de la conscience professionnelle - cette conscience qu'on croyait «avoir pour soi» - s'affrontent entre eux. Les uns ne peuvent plus «passer» par les autres, se « réfléchir» dans les autres, se réaliser au travers les autres. C'est peut-être là le nœud inconscient du drame ${ }^{3}$.

Comme l'a vu Canguilhem, on peut désespérer alors d'une société où, pour le sujet, c'est l'humanité à travers lui qui a été trahie, où les valeurs n'ont plus de valeur, où ce qui arrive peut tout simplement avoir lieu, comme si rien n'avait été vécu de ce qui pourtant a existé. Pour le sujet c'est un déchirement de la conscience qu'opère cette effraction du réel dans un professionnalisme jusque-là inébranlable. Cet «emportement» auquel il avait consenti faute de mieux, objet de tout son investissement professionnel est indétachable de lui. Dans le suicide il l'emmène avec lui, peut-être pour s'en délivrer. B. Doray a su montrer il y a déjà longtemps, avec le doigté clinique qui caractérise ses recherches, qu'à ne «faire qu'un » avec son travail on pouvait être tenté, devant des événements exceptionnels, de "disparaître avec lui » (2011, p. 164). Et le même Canguilhem de citer P. Valéry pour qui le meurtre de soi-même imposé par ce type de circonstances «procède de l'impuissance où l'on se trouve d'abolir exactement un certain mal. On ne peut atteindre la partie que par le détour de la suppression du tout. On supprime l'ensemble et l'avenir pour supprimer le détail et le présent. On supprime la conscience, parce que l'on ne sait pas supprimer telle pensée (...). Le désespéré est conduit ou contraint à agir indistinctement » (1933, pp. 71-72).

Bien sûr, il manquerait un maillon essentiel à l'analyse si on se limitait à ce brouillage ravageur dans la conscience professionnelle entre les voix impersonnelles et personnelles au cours du dialogue intérieur. Il y manquerait sans aucun doute ce qui achève de faire basculer la situation et enfante l'idée du suicide «comme une délivrance» pour le sujet (Dejours, 2010 , p. 159). Dans un tout autre contexte, N. Zaltzman a montré qu'à ce moment-là, « sa seule maladie est qu'il n'a pas âme qui vive à qui parler» (1998, p. 191). Car le désir de parler du piège d'impuissance qui s'est refermé sur le désespéré se heurte chez lui à une déception sévère. En effet, il se retourne alors contre les formes établies d'un «contact social » déréglé. Or, dans son entourage, pour ses collègues en particulier, sans répondant collectif pour défaire ces contrefaçons impersonnelles du «contact social», l'importance qu'il donne maintenant à ces contrefaçons est vaguement inquiétante. Loin d'en faire l'occasion d'une délibération collective sur la solitude dans laquelle chacun vit la paralysie de la conscience professionnelle, on n'est pas loin - à l'opposé donc de toute élaboration transpersonnelle - de considérer son intransigeance nouvelle comme un symptôme personnel suspect. Et en mettant le «suspect» un tant soit peu à l'écart pour se défendre ensemble du symptôme, on le rend - sans intention de nuire - conforme au symptôme qu'on lui prête.

Après avoir sans doute commencé - à tort ou à raison - à désespérer de l'humanité dans l'organisation, puis à s'attribuer la responsabilité d'avoir cru possible de ne «faire qu'un » avec son travail, on imagine sans doute pouvoir compter sur ses collègues pour vérifier que ce qu'on vit est bien invivable pour tous. La perte de cette dernière illusion peut suffire à faire basculer les choses. Le suicide au travail est alors l'ultime signe d'une vitalité désespérée. Peut-être même un appétit de vivre qui s'acharne contre lui-même. Il a fallu se rendre à l'évidence tragique que les registres interpersonnels et transpersonnels de la conscience ne pouvaient plus s'interposer entre soi et soi. Car, en situation, ils ne s'interposaient déjà plus entre les uns et les autres dans la conscience professionnelle collective où le discours convenu oblitère désormais les conflits. Le passage à l'acte peut donc paradoxalement suivre une prise de conscience et pas forcément la perte de cette dernière. C'est là ce qu'on propose d'appeler le drame de la conscience professionnelle.

3 On retrouve ici l'une des conceptualisations de l'inconscient proposées par Vygotski (2003, p. 78). 


\section{9.- Ne pas tricher avec le réel : la valeur du conflit}

On le voit, l'hypothèse que nous retenons est celle d'un faux mouvement de la conscience professionnelle du sujet, d'une torsion de cette dernière dans l'activité. Elle la met en contravention avec le réel. Devant un événement chaque fois singulier, impensable pour lui, l'affect fait perdre au sujet la contenance «forcée » qu'il avait chèrement acquise dans son travail. Sans espoir de pouvoir - à tort ou à raison - refaire cette contenance à court terme avec les autres en reprenant ensemble le travail d'organisation, celle ou celui qui se suicide au travail la fait disparaître avec lui. Dans ce cadre, une antinomie fréquente dans les débats en cours, qu'ils soient sociaux ou scientifiques, se trouve questionnée. En matière de causalité, l'origine personnelle ou organisationnelle des suicides au travail n'est pas directe.

La dimension personnelle de l'acte ne peut pas être effacée, ne serait-ce que par respect pour cet acte, respect auquel G. Canguilhem nous convie à juste titre. Mais la faille si souvent invoquée n'est pas à chercher en première intention dans une histoire personnelle protopathologique indépendante de la situation de travail. Elle est au cœur de la conscience professionnelle présente du sujet pris au piège d'une conscience collective dégradée dans l'organisation du travail. C'est ce piège organisé sur le simulacre du travail bien fait recouvrant en fait une conception monologique de la performance et un professionnalisme gâché - que les suicides «vindicatifs» veulent aussi, tragiquement, rendre public. L'interfonctionnalité possible ou empêchée des registres de la conscience professionnelle est d'abord sociale et organisationnelle. En activité, ces discordances sont le transformateur social qui alimente en énergie conflictuelle la conscience professionnelle personnelle. Privée de cette énergie, cette dernière se régénère mal, ne se « recharge » plus. Soustraite au jeu des comparaisons sociales, elle se « décharge » sur des buts personnels «arrêtés ». L'individuel est bien alors la forme supérieure du social comme l'écrit Vygotski mais, quand celui-ci se défait ainsi, les préjudices subis, à l'échelle d'une vie singulière, peuvent aussi être supérieurs et même, comme ici, dramatiques. On comprend alors la portée, dans la question qui nous occupe, de ce que Wallon tenait pour essentiel dans le développement du sujet: « ce qui lui est nécessaire ce n'est pas un progrès, c'est un retrait de sociabilité. Il doit pouvoir se ressaisir vis-à-vis d'autrui. Cette délimitation, loin de commander l'évolution, ne peut en être que l'effet» (1970, p. 92). C'est cette délimitation que le monologue social et organisationnel compromet. Et quand le collectif lui-même ou encore la vie hors travail ne permettent plus, parallèlement, de se démarquer pour se reprendre, le péril n'est pas loin. C'est pourquoi ces drames de la conscience professionnelle, souvent décrits comme des conflits de valeurs témoignent surtout de la valeur des conflits. Ils nous parlent des conflits qu'il vaut mieux assumer dans la délibération collective et sociale sur la qualité du travail (Clot, 2010 ; Petit, Dugué, \& Daniellou, 2011) pour que chaque travailleur s'y retrouve : pour qu'il puisse enrichir ceux auxquels sa conscience professionnelle propre ne peut échapper sans risque. L'institution du conflit dialogique est encore le meilleur moyen qu'on connaisse pour ne pas tricher avec le réel. La santé et l'efficacité en dépendent sûrement. Rien ne condamne d'avance le travail à rendre la vie impossible alors même qu'il se prête particulièrement bien à ce que chacun devienne unique en son genre.

On terminera par une dernière remarque sur l'action du psychologue du travail et de l'intervenant en santé au travail de façon plus générale : chaque fois que le monologue sur la performance recule et que progressent le dialogue et la coopération conflictuelle pour la définir, la vitalité de la conscience professionnelle collective y gagne avec la qualité du travail. Et c'est dans cette institution du conflit que se trouve sans doute le cœur du métier en clinique du travail. Dans cet article on a voulu pourtant faire un pas de plus : cette démarche peut avoir en effet une vertu thérapeutique spécifique dans le dialogue singulier qui s'installe de fait souvent entre un sujet et le psychologue du travail dans une situation dégradée. La clinique (Clot, 2002) peut consister alors, dans ce cadre-là, à accroître le seuil de tolérance dont dispose le sujet à ses propres conflits en lui permettant - autant que faire se peut - de 
se ressaisir de ceux qui ne lui appartiennent pas personnellement pour s'en différencier et s'en dégager autant que possible. En remettant le travailleur concerné en activité sur les conflits actuels de la conscience professionnelle collective dans lesquels il est emmaillé, la psychologie du travail consiste ici à aménager un autre «contact social » avec soi-même. Elle cherche à interrompre le monologue de la conscience professionnelle où se noue le drame pour soi ${ }^{4}$. Et ce, afin d'être plus soi-même avec cet intercalaire social revisité. Certes le sujet pourra d'autant plus y loger ses propres desseins et sa propre sensibilité (Davezies, Deveaux \& Torres, 2006 ; Fernandez, 2009) que ce contact social sera devenu l'objet d'une action et d'un dialogue collectifs. Quoi qu'il en soit, en prenant un peu plus personnellement possession de cet intercalaire, de ses déséquilibres inéliminables, il pourra éventuellement s'y rétablir.

\section{BIBLIOGRAPHIE}

Baudelot, C., \& Establet, R. (2006). Suicide. L'envers de notre monde. Paris: Seuil.

Barlet, B., \& Marichalar, P. (2012). Suicide. In A. Bévort, A. Jobert, M. Lallement, \& A. Mias (Eds.), Dictionnaire du travail (pp. 744-750). Paris: PUF.

Canguilhem, G. (2011). Écrits philosophiques et politiques. Euvres complètes tome I. Paris: Vrin.

Clot, Y. (2002). Cliniques du travail et psychopathologie du travail. Cliniques méditerranéennes, 66, $5-11$.

Clot, Y. (2003). La conscience comme liaison. In L. Vygotski, Conscience, inconscient, émotions. Paris: La Dispute.

Clot, Y. (2008). Travail et pouvoir d'agir. Paris: PUF.

Clot, Y. (2009). L'apport de F. Tosquelles à la clinique du travail. Postface à F. Tosquelles, Le travail thérapeutique en psychiatrie. Toulouse: Erès.

Clot, Y. (2010). Le travail à cœur. Pour en finir avec les risques psychosociaux. Paris: La Découverte.

Davezies, P., Deveaux, A., \& Torrès, C. (2006). Repères pour une clinique médicale du travail. Archives des maladies professionnelles, 67(2), 119-128.

Dejours, C. (2007). Une nouvelle forme d'aliénation qui tue. Santé \& Travail., $n^{\circ} 60,2-28$.

Dejours, C. (2010). Nouvelles formes de servitude et suicide. In C. Dejours (Ed.), Observations cliniques en psychopathologie du travail (pp. 131-161). Paris: PUF.

Dejours, C., \& Bègue F. (2009). Suicide et travail : que faire ? Paris: PUF.

Doray, B. (2011). Psychopathologie du travail. De la resymbolisation. Toulouse: Erès.

Fernandez, G. (2009). Soigner le travail. Toulouse: Erès.

Halbwachs, M. (1955). Esquisse d'une psychologie des classes sociales. Paris: Librairie Marcel Rivière.

Kostulski, K, Clot, Y., Litim, M., \& Plateau, S. (2011). L'horizon incertain de la transformation en clinique de l'activité : une intervention dans le champ de l'éducation surveillée. Activités, 8(1), $129-145$.

Laval, G. (2002). Homo analyticus, homme du politique. In A. Le Guen, G. Pragier, \& I. ReissSchimmel (Eds.). Freud, le sujet social (pp. 55-72). Paris: PUF, collection Monographies de psychanalyse.

Le Guillant, L. (2006). Le drame humain du travail. Toulouse: Erès.

Lhuilier, D. (2009). Dépressions sévères et travail. L'Encéphale, Supplément, X, SXX, 51-55.

Lhuilier, D. (2011). Suicide et travail. La revue du praticien, 61, 198-201.

Malrieu, Ph. (2013). Les fonctions sociales de la psychologie. In A. Baubion-Broye, R. Dupuy, \& Y Prêteur (Eds.), Penser la socialisation en psychologie. Actualité de l'œuvre de Philippe Malrieu. Toulouse: Erès.

4 Dans un prochain article je me propose de revenir sur les développements de l'activité transférentielle impliquée par l'exercice : Affects, émotions et sentiments en psychologie du travail (à paraître). 
Maggi, B. (Ed.). (2011). Interpréter l'agir. Un défi théorique. Paris: PUF.

Molinié, A.-F., Gaudart, C., \& Pueyo, V. (Eds.). (2012). La vie professionnelle : âge, expérience et santé à l'épreuve des conditions de travail. Toulouse: Octarès, Collection « Travail et Activité humaine $»$.

Molinier, P. (2009). De la rumeur à la peur. Une enquête de psychodynamique du travail à la suite d'un suicide lié au travail. Communication et organisation, 36, 44-54.

Petit, J., Dugué, B., \& Daniellou F. (2011). L’intervention ergonomique sur les risques psychosociaux dans les organisations : enjeux théoriques et méthodologiques, Le Travail Humain 74(4), 391-409.

Scheller, L. (2002). Clinique de l'activité, haine, travail. Cliniques méditerranéennes, 66, 85-105.

Terssac, de, G. (2011). Théorie du travail d'organisation. In B. Maggi (Ed.). Interpréter l'agir. Un défi théorique (pp. 97-124). Paris : PUF.

Tosquelles, F. (1984). Éducation et psychothérapie institutionnelles. Hiatus Edition.

Valéry, P. (1933). Rhumbs. Paris: Gallimard.

Vygotski, L. (1997). Pensée et langage. Paris: La Dispute.

Vygotski, L. (2003). La conscience comme problème de la psychologie du comportement. In L. Vygotski, Conscience, inconscient, émotions. Paris: La Dispute.

Vygotski, L. (2004). Psychologie concrète de l'homme. In M. Brossard (Ed.), Lectures et perspectives des recherches en éducation. Lille: Septentrion.

Wallon, H. (1970). De l'acte à la pensée. Paris: Flammarion.

Wallon, H. (1971). L'étude psychologique et sociologique de l'enfant. Enfance, numéro spécial Henri Wallon.

Zaltzman, N. (1998). De la guérison psychanalytique. Paris: PUF.

\section{RESUME}

L'article propose une hypothèse de travail sur les suicides en milieu professionnel. À partir des travaux de Vygotski en théorie de l'activité et de l'analyse de G. Canguilhem sur la conscience, il discute les résultats de la sociologie sur les suicides. Il expose les débats en cours en psychopathologie du travail. Il développe la conception selon laquelle les suicides au travail sont des drames de la conscience professionnelle. C'est la confusion entre conscience collective et conscience personnelle qu'il faut étudier pour mieux comprendre les rapports entre individu et organisation du travail qui sont au coeur du problème.

\section{MOTS CLES}

suicides en milieu professionnel, drames de la conscience professionnelle, psychopathologie du travail, Vygotski.

\section{REFERENCEMENT}

Clot, Y. (2013). Suicides au travail : un drame de la conscience professionnelle ? Activités, 10(2), 39-53, http://www .activites.org/v10n2/v10n2.pdf

Article soumis le 19 avril 2013, accepté pour publication le 14 septembre 2013 